\title{
Preface to the VECoS 2018 special issue of ISSE
}

\author{
Mohamed Faouzi Atig ${ }^{1}$ Simon Bliudze ${ }^{2}$
}

Published online: 30 May 2020

(c) Springer-Verlag London Ltd., part of Springer Nature 2020

This special issue contains the extended versions of selected papers from the 12th International Conference on Verification and Evaluation of Computer and Communication Systems (VECoS 2018) held during September 25-28, 2018, at Université Grenoble Alpes in Grenoble, France.

The aim of the VECoS conference is to bring together researchers and practitioners in the areas of verification, control, performance, and dependability evaluation in order to discuss the state of the art and challenges in modern computer and communication systems in which functional and extra-functional properties are strongly interrelated. Thus, the main motivation for VECoS is to encourage cross-fertilisation between various formal verification and evaluation approaches, methods, and techniques, and especially those developed for concurrent and distributed hardware/software systems.

This issue comprises 7 papers covering various aspects of computer and communication systems verification and evaluation: 2 papers focus on testing techniques, 3 contribute to the domain of model checking, 1 paper proposes a synthesis approach, and 1 paper reports on the analysis of a transportation system.

- The paper "Combined software and hardware fault injection vulnerability detection" by Given-Wilson et al. [1] aims at locating potentially security-relevant fault-injection vulnerabilities in software running on micro-controllers. The proposed approach is intended to detect machine-code constructs that can be targeted with hardware fault-injection (FI) methods by an attacker with physical hardware access, achieving a deviating behaviour with security implications. The

Simon Bliudze

simon.bliudze@inria.fr

Mohamed Faouzi Atig

mohamed_faouzi.atig@it.uu.se

1 Department of Information Technology, Uppsala University, Uppsala, Sweden

2 Inria Lille - Nord Europe, Lille, France authors conduct simulation-based FI experiments and hardware-implemented ones inducing faults by elecrtomagnetic pulse (EMP) on two micro-benchmarks. They demonstrate that a subset of their fault models in the simulation-based FI locates all vulnerabilities but also several false positives. Conclusions include insights on how to appropriately model EMP faults, and that a combination of simulation-based and hardware-implemented FI can demonstrate genuine FI vulnerabilities with significantly reduced effort compared to a purely hardwarebased approach.

- The paper "How to be sure a faulty system does not always appear healthy?" by Dague et al. [2] studies the notion of manifestability for discrete event systems prone to failures modelled as finite or timed automata. Manifestability is a weak variant of diagnosability whereby one requires that at least some - as opposed to every, for diagnosability - faulty behaviour can be detected. The authors show that this problem is PSPACE-complete for finite automata, undecidable for timed automata, and PSPACE-complete again for a subclass of timed automata. They also give experimental results and, for timed automata, explain how to efficiently translate the problem in view of an implementation.

- The paper "Improving swarming using genetic algorithms" by Renault [3] presents a novel swarming approach for parallelizing depth-first search (DFS) graph exploration algorithms by combining classical swarm algorithms and genetic programming. The approach is illustrated for checking safety properties, and an indication is given on how to use it for liveness properties also (emptiness checks for Büchi automata). The main idea is that some of the threads start the exploration from artificial initial states inside a super-set of the reachability set. The intuition behind this idea is that they are possibly inside the reachability set and in the good cases closer to a deadlock state or an accepting cycle. The approach has been evaluated on 38 models from the BEEM suite (BEnchmarks for Explicit Model checkers). 
- The paper "LTL model checking for communicating concurrent programs" by Pommellet and Touili [4] studies the model checking problem for single-indexed Linear Temporal Logic (LTL) properties on communicating push-down systems (CPDSs). Single-indexed LTL properties are conjunctions of local properties, one for each component. The authors propose to use abstract interpretation to abstract the single components and approximate the set of traces satisfying the property. If the intersection of the over-approximations of the components is empty, it can be concluded that the property cannot be satisfied. Otherwise, the intersection should be analysed to see if there are concrete traces. However, the possibility of automating this analysis remains an open problem.

- The paper "Exploiting local persistency for reduced state space generation" by Barkaoui et al. [5] deals with the problem of the state space dimension in model checking Petri Nets by investigating partial order techniques based on persistent sets and step graphs methods. In particular, it proposes the persistent step sets as a parametric combination of both methods in order to compute covering steps, persistent sets, persistent-step sets and other kinds of combination of both methods. Two algorithms for the computation of persistent step sets using strongpersistent sets and weak-persistent sets, respectively, are presented, together with an analysis of how to weaken the sufficient conditions of persistent sets. The introduced algorithms are also empirically evaluated.

- The paper "Timed service contract automata" by Basile et al. [6] introduces a new formal model, Timed Service Contract Automata (TSCA), to rigorously reason about the creation, matching (offer, request, agreement), and composition (orchestration) of services that are also subject to real-time constraints in addition to various levels of criticality. Specifically, the authors provide conditions for TSCA composability and methods to compute their composition. They introduce the notion of semi-controllability which becomes necessary to avoid "dangling configurations", i.e. configurations that are not reachable or from which no final state can be reached. In addition to supervisory control theory, concepts from timed games are utilised to develop the orchestration synthesis algorithm. The results are applied to the example of a hotel reservation system by computing the winning strategy by which both the reservation desk and the customer can achieve their goals and the automaton enters a success state.
- The paper "Stochastic modeling and analysis of roadtramway intersections" by Carnevali et al. [7] presents a use case for stochastic modeling and analysis using stochastic timed Petri nets. The use case is about critical intersections where several kinds of traffic are mixed, namely public tramway traffic and private cars traffic. Modeling and analysis of such intersections allow the measurement of relevant traffic characteristics such as probabilities of traffic congestion, average waiting time in queues, and recovery times. This contributes to a better understanding of the impact of various parameters (tram schedules, traffic light periods, etc.) on the overall traffic behaviour. These results can be leveraged for organising and adapting the traffic in real traffic situations.

We would like to thank all the authors of these papers for their contributions and the reviewers that we have solicited for their thorough evaluations. We are particularly grateful to the ISSE editor-in-chief Mike Hinchey and the editorial assistant Chitra Vijayaraghavan for their help and reactivity throughout the preparation of the issue.

\section{References}

1. Given-Wilson T, Jafri N, Legay A (2020) Combined software and hardware fault injection vulnerability detection. Innov Syst Softw Eng 16(2/3). https://doi.org/10.1007/s11334-020-00364-5

2. Dague P, He L, Ye L (2020) How to be sure a faulty system does not always appear healthy? Fault manifestability analysis for discrete event and timed systems. Innov Syst Softw Eng 16(2/3). https://doi. org/10.1007/s11334-019-00357-Z

3. Renault E (2020) Improving swarming using genetic algorithms. Innov Syst Softw Eng 16(2/3). https://doi.org/10.1007/s11334-02000362-7

4. Pommellet A, Touili T (2020) LTL model checking for communicating concurrent programs. Innov Syst Softw Eng 16(2/3). https:// doi.org/10.1007/s11334-020-00363-6

5. Barkaoui K, Boucheneb H, Li Z (2020) Exploiting local persistency for reduced state space generation. Innov Syst Softw Eng 16(2/3)

6. Basile D, ter Beek M H, Legay A (2020) Timed service contract automata. Innov Syst Softw Eng 16(2/3). https://doi.org/10.1007/ s11334-019-00353-3

7. Carnevali L, Fantechi A, Gori G, Vicario E (2020) Stochastic modeling and analysis of road-tramway intersections. Innov Syst Softw Eng 16(2/3). https://doi.org/10.1007/s11334-019-00355-1

Publisher's Note Springer Nature remains neutral with regard to jurisdictional claims in published maps and institutional affiliations. 